\title{
Intelligent Framework for Public Transport Bus Services system
}

\author{
Nithesh Chandher Karthikeyan, Aswath Ananthasamy, Yokesh babu Sundaresan
}

\begin{abstract}
The word "Smart City" has been invented by International Business Machines Corporation's "Smart Planet" through an application from a new variety of information, with life more refined and dynamic. Smart City is the future of our country with transportation being one of the main features designed to make a city smart. In India, the automotive sector in India is one of the main contributors of the nation's GDP, thus representing its importance. In this survey paper, we have discussed the actual meaning of a smart city and an important feature of a smart city, which is, smart transportation present in the city. We have discussed about various important smart features which are needed for the transportation sector. We have also compared those features of how they have been implemented in some International cities.
\end{abstract}

Index Terms: About four key words or phrases in alphabetical order, separated by commas.

\section{INTRODUCTION}

The word "Smart City" has been invented by International Business Machines Corporation's "Smart Planet" through an application from a new variety of information, with life more refined and dynamic [1]. Smart cities speak to an applied urban improvement show in light of the use of people, aggregate, and mechanical income for the upgrade of improvement and thriving in urban mass. In any case, vital making arrangements for smart city improvement still remains a fairly conceptual thought for a few reasons, counting the way that it alludes to so far to a great extent unexplored what's more, interdisciplinary fields [2]. Building a city "smart" is creating as a system to ease the issues delivered by the urban people improvement and quick urbanization. In light of the investigation of a wide and expansive group of composing from various disciplinary areas there are eight perceived fundamental factors of smart city exercises: the board and association, innovation, administration, arrangement setting, individuals and networks, economy, assembled framework, and peaceful environment. These components shape the introduce of an integrative framework that can be utilized to take a gander at how neighborhood governments are envisioning smart city exercises. The structure suggests inspiration for smart city research and systems helpful consequences for government specialists [3]. Digital urban areas have been progressed from web applications and data bases to smart urban circumstances. This progression has mainly been established on broadband metro-systems and complex data frameworks,

Revised Manuscript Received on July 05, 2019.

Nithesh Chandher Karthikeyan, Student, Computer Science and Engineering, Vellore Institute of Technology, Vellore.

Aswath Ananthasamy, Student, Computer Science and Engineering, Vellore Institute of Technology, Vellore.

Yokesh Babu Sundaresan, Professor, Computer Science and Engineering, Vellore Institute of Technology, Vellore. and it prescribes the kind of things to come city that is called digital city [4].

I IMPORTANCE OF TRANSPORT IN A CITY

\section{A. Economic Role [5]}

$>$ Transport broadens the scope of supply of supply of merchandise to be expended in a region, making it feasible for user to get assets at less cost and high quality.

$>$ The utilization of progressively proficient systems of supply results in an expansion in the total sum of products available for consumption.

\section{B. Social Role of Transportation \\ $>$ Formation of settlements. \\ $>$ Growth of size of cities. \\ C. Political Role of Transportation \\ $>$ Administration of an area (Sending information to/ from the people). \\ $>$ Different types of transport such as communication, military. \\ D. Environmental role of Transportation \\ $>$ Safety}

\section{SMART TRANSPORTATION}

Smart Transportation is one of the main features of Smart Cities. It gives an efficient way to handle the functioning of mobility in cities. In $1950,30 \%$ of the total populace was urban. This rate is required to develop to 66 percent by 2050 . The European Commission is focused on enhancing the personal satisfaction in urban communities and economic development through the advancement of transport arrangements that are economical, energy efficient and environment free [6]. Transport request in the greater part of the Indian urban communities has expanded significantly because of the expansion in the populace as a result of both common increment and movement from rural zones and residential areas. Accessibility of motorized vehicles, increments in family salary, and an expansion in business and industrial activities have additionally added to it. Tragically, public transport frameworks in Indian urban communities have not possessed the capacity to keep pace with the quick and significant increment in travel request. Rail-based public transport administrations and efficient transport administrations are constrained only to couple of huge urban communities. The issue that is mentioned is one of the problems that are faced in the transportation sector in India. These issues in transportation is increasing in India day by day as the automotive sector in India is one of the main

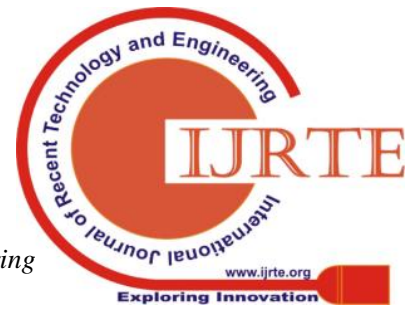


contributors of the nation's GDP [7].There are many challenges that are faced in Smart Transportation in the global context like Unorganized car parking, Inadequate public transport, Vehicle tracking systems for public transport, Transport emission and air quality monitoring, Effective toll fee collection, Unawareness about the schedule of public transport, ticket fare collection in a crowded bus and many more. In this paper, we will address the general problems that are faced in a public transport, traffic signals and parking systems that are listed below.

1) Smart Ticket Fare Collection

2) Public Transport Tracking

3) Customer Behaviour

4) Smart Priority Traffic Signal

5) E-parking System.

6) Safety

\section{Smart Ticket Fare Collection}

\subsection{Challenges}

Usually we can witness in the public transportation that the conductors confront many issues in gathering the fare from the passengers. Cash taking care of and restoring the overabundance add up to the passengers in the wake of deducting the fare sum, assessing a travel pass winds up complex when the congestion (that is the number of passengers is vast in numbers) is high and this unpredictability can't be overseen viably. Profitability in urban places is much subject to how effective the vehicle framework is to move a traveling bunch from different sources to various terminus. Also, in bus terminus, people are unaware about the schedule of buses to their destination and the public is really struggling to plan their journey. People are also unaware of the availability of buses to their destination as the transportation authority finds it difficult to manage the crowd. Because of this issue, some buses have vacant seats and other buses are highly populated even though they are going to reach the same destination [8].

\subsection{Generic Work Flow Solution}

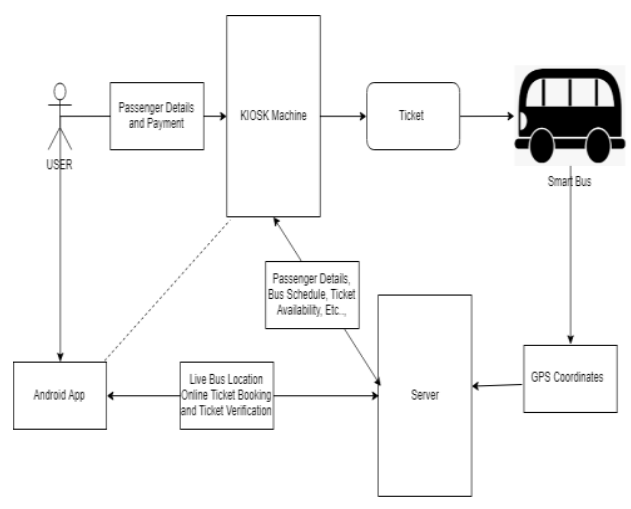

Fig 1. Work Flow of Smart Ticket Fare Collection

1.3 Existing Solution in Stockholm

1. Tickets for your Journey with SL:

The passengers are given an electronic smart card which is known as the SL smart card, which contains almost all the passenger's tickets for travel.

\section{Scan in when you travel:}

In each metro stations, there are computerized barriers with blue readers which scans your SL Access card for the availability of ticket in your name and allows you to board the train or bus.

3. Pay in advance for your journey:

It is always advisable to purchase your tickets in advance as it is a prepaid card, which is cheaper for longevity. For temporary travels, it is best to buy single visit cards or book via the smart mobile app as it will suit our purpose much better [9].

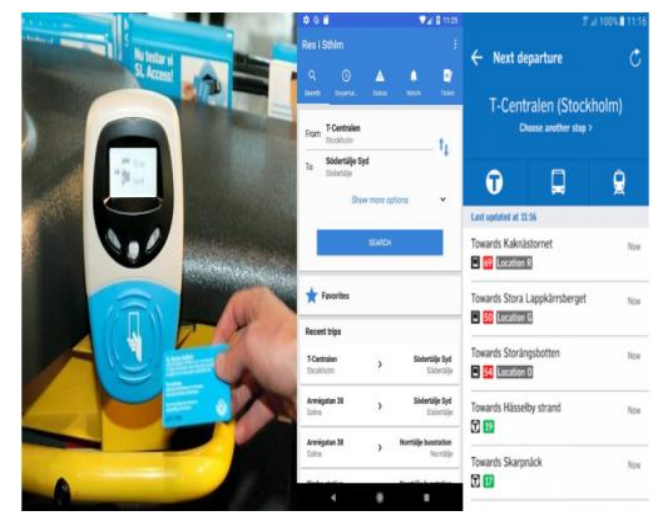

Fig 2. Case Study (City of Stockholm)

\subsection{Proposed Solution}

We can provide a mobile application-based ticket fare collection system in which the public can register their identity in order to activate an account through which payments can be done to avoid the main problem of complicated ticket fare collection system. Also, we can update the passengers about the timings and availability of buses which can help them to plan their trip in advance [10]. Dataset:

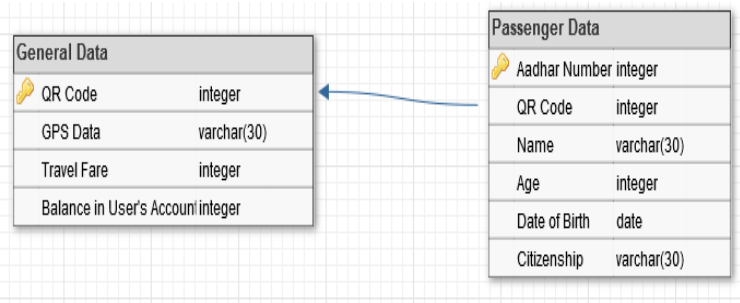

Fig 3. Relational Schema of Smart Ticket Fares Collection QR Code is used for authenticating the ticket confirmation for the passenger. For the live tracking of the transports, the GPS data is used. The Travel Fare indicates the price for each journey, while the Balance in User's account reveals how much money the passenger still has left in his account. The Aadhar Number of the passenger, the name, age, date of birth and citizenship are collected from the passenger for their identity and to determine consumer behaviour.

\section{Public Transport Tracking}

\subsection{Challenges}

The main problem with using a public transport in India is being completely unaware about its arrival to its destination. Hence, a lot of people's time and energy are

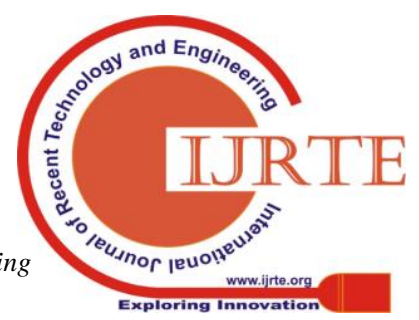


wasted, also increases the crowd and a sense of confusion among the people who want to use the public transports.

\subsection{Generic Work Flow Solution}

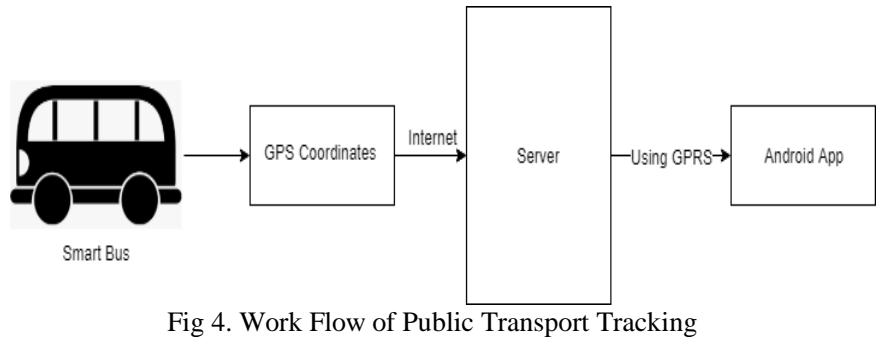

\subsection{Existing Solution in Barcelona}

The metro in Barcelona is a standout amongst the most present day and best structured frameworks in the world. The metro tracking systems are done with GPS and data are sent to the people using GPRS primarily. There is a metro map online which can be downloaded by the passengers which can be used to track the metro in real time, all the time. In bus stations, there are solar powered touch screen boards which can also be used to view the location of a bus, and its estimated time of arrival and the number of passengers that are on board of the bus [11].

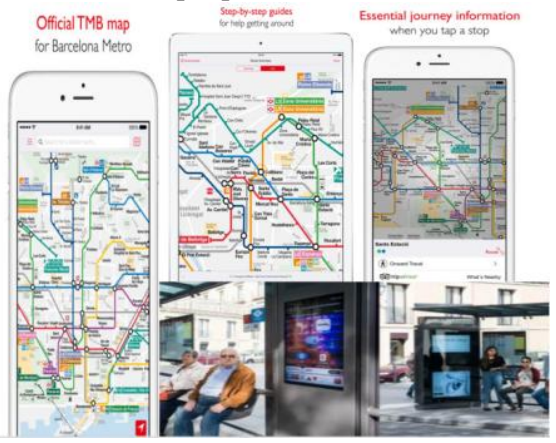

Fig 5. Case Study (City of Barcelona)

\subsection{Proposed Solution}

Our solution for an Automatic Vehicle Location Systems is to automatically locate a vehicle with the help of a GPS device, send the location to a main server via the internet and send the details to the passengers using a GPRS device [12].

The Technologies used are: -

- GPS

- GRPS (to connect mobile with internet)

- Server

- Workstation

The Dataset necessary for an AVLS are: -

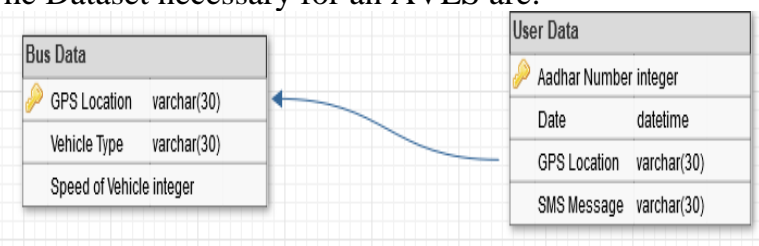

Fig 6. Relational Schema of Public Transport Tracking

The GPS location data is used for live tracking of the transports. The medium of transport is determined through the vehicle type, and the duration to the destination is determined through the speed of vehicle. The passenger receives the GPS location through SMS message acting as a medium, with the Date field providing the live time and the Aadhar number is a unique number for every passenger.

\section{Customer Behaviour}

\subsection{Challenges}

It is really difficult to predict the public behaviour in any cities especially in the field of transportation. Urban people are living a life in a scheduled manner and transportation comes as a part in their schedule. Now there comes a situation where a people of a particular category use the public transport during a particular period of time and their needs should be satisfied by the transportation authority by allocating the right number of buses in order to accommodate those passengers. Hence the unpredictability of the user behaviour is a major issue and is one of the reasons behind chaos during peak hours due to unavailability of public transportation to reach their destination.

\subsection{Generic Work Flow Solution}

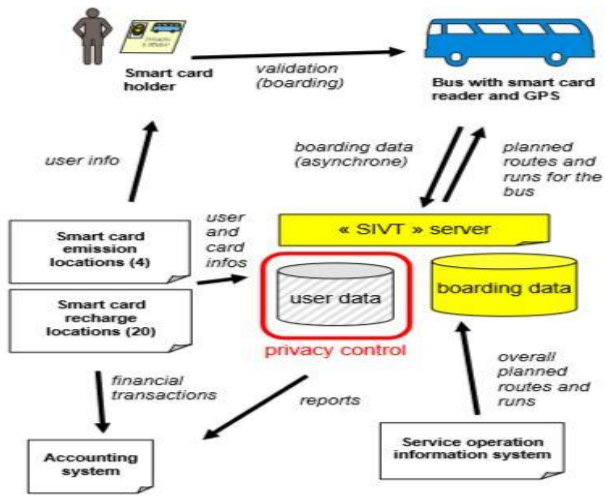

Fig 7. Work Flow of Customer Behaviour [13]

\subsection{Existing Solution}

Catching individuals' personal behaviour standards, for example, buy patterns or shopping behaviour standards, are huge promoting worry since such patterns are great assets to create new showcasing services. To this end, a great deal of studies utilizing POS (Points of Sales) information have been led to extricate buy practices for CRM (Customer Relation Management). Moreover, since a great deal of boundless sensor devices, smart cards or cell phones have turned out to be accessible as of late, anybody can attempt to analyse pedestrians' directions got from GPS information and concentrate individuals' personal behaviour standards. Besides, with the spread of smart IC cards, analysis of utilization chronicles of the cards has likewise pulled a lot of consideration nowadays.

Investigating buy practices in the wake of getting off the train at each station in Japan, we can gauge buy conduct tendency at shop affiliations in some specific schedule opening, the unpleasant separation between the shops and the station, conditions around stations, and their time-variable usage [14]. 3.4. Findings

1) The buy patterns are done with a standard time of couple of minutes after the arrival of the train.

2) The buy patterns to parking lot is usually collected within ten minutes after the arrival of the train. This implies the parking lot users by smart IC card are situated around station.

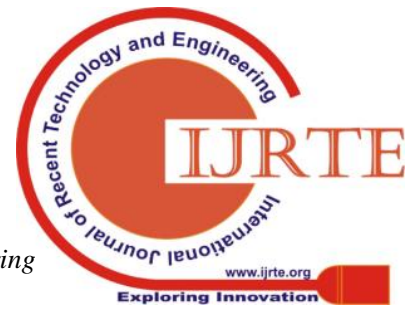


3) Different markets are used by people after the arrival of the train depending on its arrival time which is nothing but the length to the receipt counter.

4) The buy behaviour patterns after arrival of the train is relatively proportional to the number of people leaving the train, which is compared with their general user behaviour. 5) In the more frequently used stations, there is a significant amount of contrast noticed between the number of people after leaving the train and those who are then shopping after arrival of train.

\subsection{Proposed Solution}

In urban public transport, client enrollment information utilizing android application is referenced of a large number of information of customers boarding vehicles over the system over a couple of days. The issue that we address is whether data mining algorithms can be used to ponder customer pattern from these discernments. This must be done with the help of transportation arranging information. While Mobile Application based Automated Fare Collection Systems' commonness is extending openly transport, a broad measure of information is gathered each day in the current structures. In spite of the way that they are essentially intended for money gathering, we can help coordinators with better learning of open transport client rehearses, thusly upgrading the administration. Without a doubt, these systems contain information on each terminus in an open transport arrange, with the particular time and some precision on a region. The datasets conveyed by these systems grow rapidly and requires capable data mining tools. It is our conviction that data mining gathers important data processing techniques to enhance the pertinence of client information.

\section{Smart Priority Traffic Signal}

\subsection{Challenges}

In the cities nowadays, there are a lot of traffic congestion on the roads. This is mainly because of improper organization of traffic signals. The traffic signals usually have a fixed time, no matter the time, the amount of traffic and it also does not consider if any emergency vehicles are travelling down the road.

\subsection{Generic Work Flow Solution}

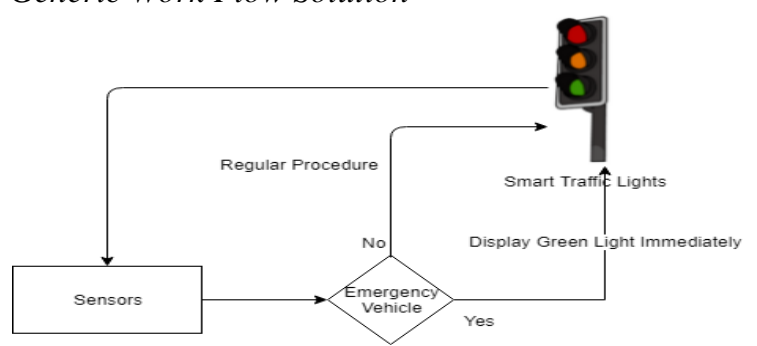

Fig 8. Work Flow of Smart Priority Traffic Signal

\subsection{Existing Solution in Barcelona}

In Barcelona, adaptive traffic signals and smart traffic signals were one of the first initiatives taken by the government in order to make their city smart. To help blind people cross the road, there is a buzzer which rings whenever the pedestrian crossing becomes green. Also, for adaptive traffic signal, when the signal becomes yellow (few seconds before becoming red), there is a sensor which scans the road for any vehicles that might need some more time to cross the road and will not change to red till that time. This sensor also scans for any emergency vehicles on the road to turn the signal green [15].

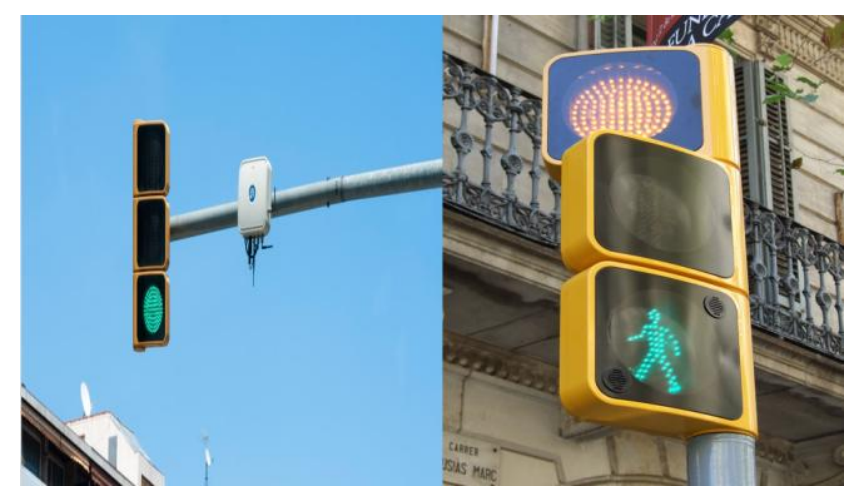

Fig 9. Case Study (City of Barcelona)

\subsection{Proposed Solution:}

We are proposing to make an adaptive traffic signal which is dynamic in nature. We use three process, the vehicle detection: to determine the amount of traffic in an area or if there is an emergency vehicle on the road, green light sequence determination: the number of signals which are needed to be converted to green, while other signals need to be converted to red and finally light length determination: to find the duration of time to change the green light to red or vice versa for a particular signal [16].

Dataset: -

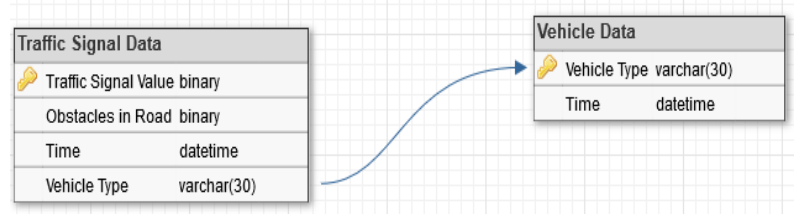

Fig 10. Relational Schema of Smart Priority Traffic Signal

The Traffic Signal Value indicates the colour of that particular traffic signal. The volume of traffic on that particular road is determined by the obstacles in road entity. The time represents live time, while the vehicle type scans the road for any emergency vehicles.

Technology Used: -

- Traffic System Server

- GPS

- GPRS

- Traffic Controller

- Sensors for Detecting Vehicles volume, effects of road $\bullet$ WIFI

\section{E - Parking}

\subsection{Challenges}

There is a high increase of use of vehicles in urban society. Many people are parking their vehicles in an improper manner, which causes a huge deal of problems to all other vehicles in that area. This is mainly due to insufficient number of parking spots, not visible areas to park vehicles and no proper guidance of how to park their vehicles in a manner which would not disturb any other vehicles.

\subsection{Generic Work Flow Solution}




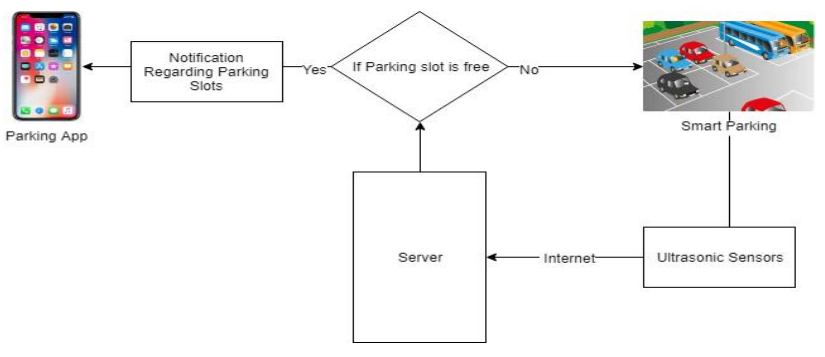

Fig 11. Work Flow of E - Parking

\subsection{Existing Solution in Barcelona}

There is an app in Barcelona called "Barcelona Locator Parking Map" which enables the user to locate a parking lot in the user's locality if he has not found one himself. This mobile application will help the user to identify the closest parking slot in all districts of the city. They should be able to find detailed information about each car park that is listed. They can also check the compare the different rates of each car parks to find the one that is right for Them. The car parking map has been divided into the different districts of Barcelona so all the user has to do is click on that neighbourhood on the map to go to a detailed view of the car parks in that zone. They can then click on the parking icons on the map to go to a detailed information page on that car park [17].

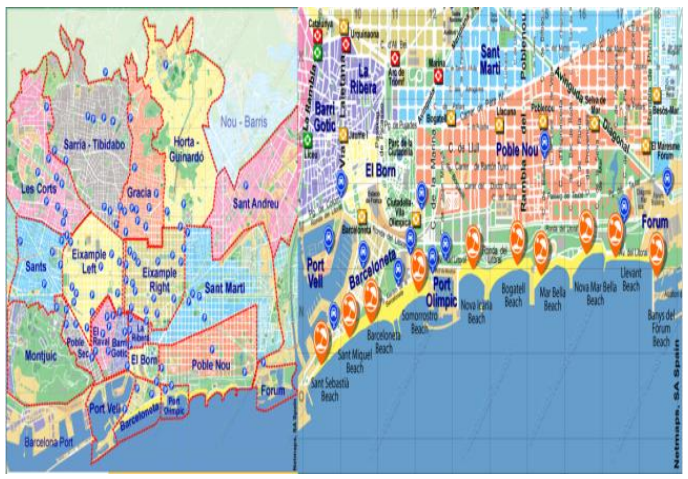

Fig 12. Case Study (City of Barcelona)

\subsection{Proposed Solution}

We have an automated smart parking management system, that would help the driver of the vehicle to locate nearby parking lots with ease. Then we deploy parking meters in parking areas with WIFI connected to a local server to monitor how a vehicle is being parked by the driver. There is a buzzer (alarm) at each parking lot that would go off if a vehicle has been improperly parked or has been parked in a wrong slot [18].

Technology Used: -

- Automatic License Plate Recognition

- PLMS

- WIFI

- Internet

- Smart Parking Meter

- For Parking Meter:

$>$ Microcontroller

$>$ Solar Panels

$>$ Ultrasonic sensor

\section{Safety}

\subsection{Challenges}

One of the main problems or challenges for the transportation of a city is the transport's safety. The safety features include both for pedestrians on the road and for the passengers travelling inside the vehicles. This has become a major concern for India with over 1,00,000 people dead because of road accidents in 2018 [19].

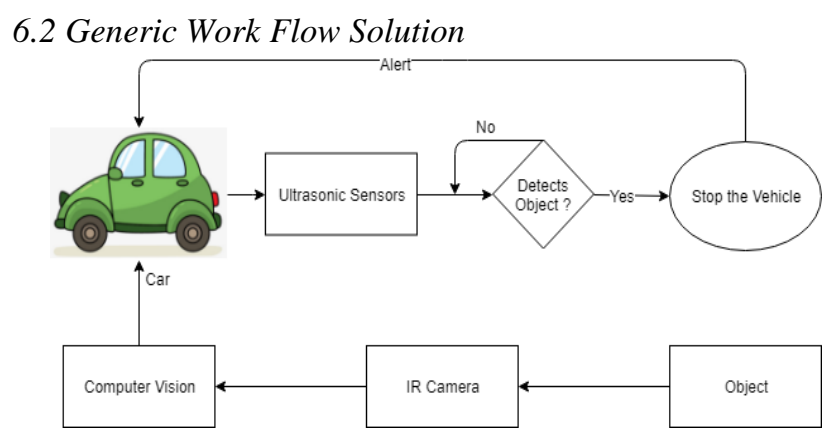

Fig 13. Work Flow of Safety

\subsection{Existing Solution in Barcelona metro}

Barcelona's metro systems are one of the most efficient and safest metro systems in the world. The metro systems have tv monitors for visual information, video surveillance for security inside the metro, intercoms for passenger-control center communication, synchronized platform doors to prevent intrusions, automatic driving trains, continuous distance measurement with destination, address of the current location, redundant equipment in case of failures, remote controls of trains from control center in case of emergencies and a 24-hour supervision from the control center.

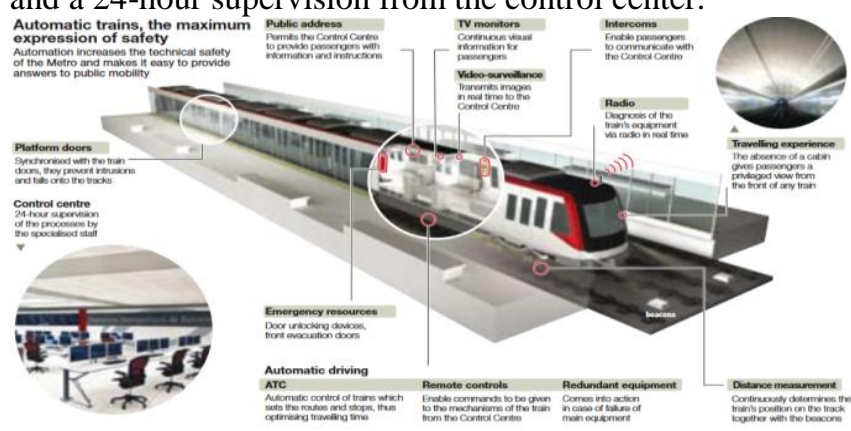

Fig 14. Case Study (City of Barcelona)

\subsection{Proposed Solution}

Our solution is to use multiple sensors placed on the road and inside the vehicles to avoid collisions with pedestrians, fellow vehicles and any other objects that might provide fatal [20]. The sensors that are used are: -

- Piezoelectric sensor (for detecting pedestrians on the road)

- Ultrasonic sensors (for detecting nearby objects/vehicles)

- Microwave radar (uses electromagnetic waves for detecting speed of nearby vehicles)

- Computer vision (vision based for making front view and back view visions easier)

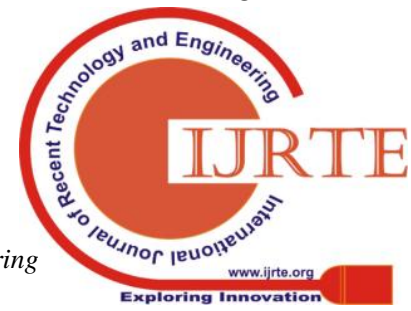


Dataset: -

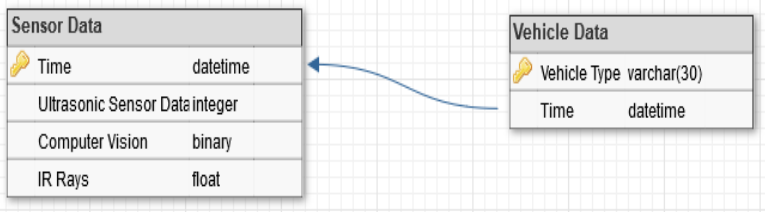

Fig 15 - Relational Schema of Safety

The time represents the live time. The ultrasonic Sensor Data, Computer Vision and IR Rays entities represents the values obtained by placing those sensors for the purpose of safety. The vehicle type represents the type of vehicle that the passenger is travelling in.

\section{OVERALL WORK FLOW DIAGRAM}

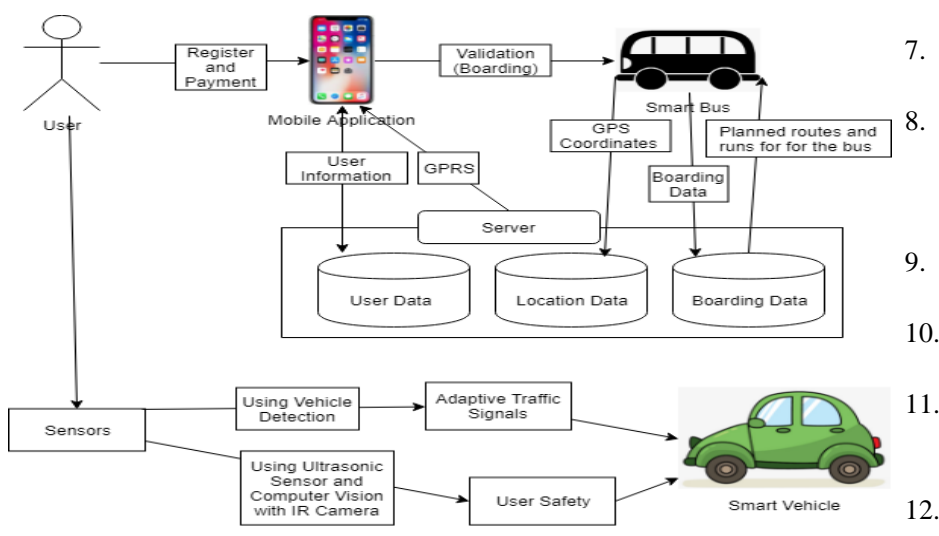

Fig 16. Overall Work Flow Diagram five features: smart ticket fare collection, public transport tracking, customer behaviour, smart priority traffic signal and safety. The user registers his detail with Aadhar number taken as a primary entity and in this way, he creates an account through which the Ticket booking and payments are taken care of. The sever is connected to 3 databases: user data contains the details of passengers, location data contains GPS coordinates of the bus, which is used for live tracking and the boarding data contains boarding information of passengers and the planned routes for the buses. We use vehicle detection using Image processing to determine if a vehicle is an emergency vehicle or not. Similarly, for the safety of the vehicles and pedestrians, various sensors are installed in the given vehicle and it alerts the driver about any unwanted collisions that might occur.

\section{CONCLUSION}

In this survey, we have discussed about the basic concepts of a smart city and have explained in detail about various important features of smart transportation in a smart city. We have explained in detail about the challenges we face when we have a city without these features. We have described a generic workflow of these features. We have also discussed about how these features have been implemented in already established smart International cities of Barcelona, Spain and

1
The above figure represents the overall work flow diagram of

Stockholm, Sweden. We have also given our proposed solution for these features.

The future work can be collection of metro dataset from Tamil Nadu Transport Department regarding ticket fares, vehicle tracking and safety measures and then implementation of our proposed solutions in the city of Vellore, India.

\section{REFERENCES}

J. Lingli, "Smart city , Smart transportation Recommendations of the logistics platform construction," 2015. M. Angelidou, "Smart city policies : A spatial approach," Cities, vol. 41, pp. S3-S11, 2014.

3. H. Chourabi, J. R. Gil-garcia, T. A. Pardo, H. J. Scholl, S. Walker and K. Nahon, "Understanding Smart Cities: An Integrative Framework," 2012.

4. L. Anthopoulos and P. Fitsilis, "From Digital to Ubiquitous Cities: Defining a Common Architecture for Urban Development."

5. Role of Transportation in Society, (2009, July 28), Retrieved from https://www.civil.iitb.ac.in/tvm/1100_LnTse/102_lnTse/plain/pla in.html

6. M. Pe, "Transportation Problems and their Potential Solutions in Smart Cities," pp. 195-199, 2017.

D. H. Mrityunjaya, N. Kumar, S. Ali, and H. M. Kelagadi, "Smart Transportation," pp. 1-5, 2017.

T. D. Camacho, M. Foth, and A. Rakotonirainy, "Pervasive Technology and Public Transport: Opportunities Beyond Telematics," 2012.[9] Fares and Tickets, Tickets for your SL Journey. Retrieved from

9. Fares and Tickets, Tickets for your SL Journey. Retrieved from https://sl.se/en/fares--tickets/

10. D. G. Chandra and R. Prakash, "Mobile Ticketing System for Automatic Fare Collection Model for Public Transport," 2013.

1. Transports Metropolitans de Barcelona, Retrieved from https://www.tmb.cat/en/barcelona/applications-downloads/tmb-a pp

B. Predić, D. Rančić, D. Stojanović, and A. Milosavljević, "Automatic Vehicle Location in Public Bus Transportation System 2 Characteristics of Vehicle Trajectories in City Public Bus Transport," pp. 675-680, 2007.

13. B. Agard and C. Morency, "Mining Public Transport User Behaviour from Smart Card Data Mining Public Transport User Behaviour from Smart Card Data Bruno Agard CIRRELT-2007-42," no. June 2017, 2006.

14. A. Kiyohiro, K. Yamaguchi, H. Gao, H. Nakamura, and T. Mine, "Customer behavior analysis on after getting off the train based on usage histories of smart IC card," 2014.

15. Mobility Services Lab, Retrieved from https://www.mobility-services.in.tum.de/?p=2237

16. T. Hong, K. Polytechnic, and H. Kong, "Adaptive Traffic Light Control in Wireless Sensor Network-based Intelligent Transportation System."

17. Tourist Guide Barcelona. Retrieved from https://www.barcelona-tourist-guide.com/en/transport/car-parkin g-barcelona.html

18. P. Sadhukhan, "An IoT-based E-Parking System for Smart Cities," pp. 1062-1066, 2017.

19. Automated Metro, Safer and more efficient, Transports Metropolitans de Barcelona. Retrieved from https://www.tmb.cat/documents/20182/111197/Dossier+metro+a utom\%C3\%A0tic+L9+angl\%C3\%A8s.pdf/1556963d-45d2-4da9 -9944-dcb470454797

20. F. Bu and C. Chan, "Pedestrian Detection in Transit Bus Application: Sensing Technologies and Safety Solutions," pp. 100-105.

\section{AUTHORS PROFILE}


Nithesh Chandher Karthikeyan Student at Vellore Institute of Technology, Vellore. Pursuing studies in Bachelors of Technology in Computer Science and Engineering.

Aswath Ananthasamy Student at Vellore Institute of Technology, Vellore. Pursuing studies in Bachelors of Technology in Computer Science and Engineering.

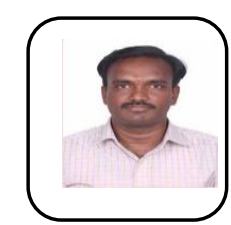

Yokesh Babu Sundaresan Professor at Vellore Institute of Technology, Vellore. In the department of Computer Science. 